\title{
A New Methodology For On-Line Power Quality Assessment
}

\author{
A. Moussa ${ }^{1}$, M. El-Gammal1, E. N. Abdallah ${ }^{1}$, and A. Abouelseoud ${ }^{2}$ \\ ${ }^{1}$ Department of Electrical Engineering \\ Alexandria University \\ Camp Chizar - Alexandria (Egypt) \\ e-mail: abouelseoud99@hotmail.com
}

\begin{abstract}
The development of new methods for real time detection and evaluation of voltage events in the distribution systems is of great importance in order to prevent the impact of these disturbances on customer equipment and to evaluate the economics of power quality improvement alternatives. The main objective of this work is to introduce a new methodology of advanced power quality assessment. The introduced system is implemented using powerful software algorithms to construct a system for real time detection and identification of different types of power quality disturbances. A number of disturbance events have been simulated for further processing and analysis The introduced system detects accurately any power quality disturbance event and introduces new indicative factors estimating the performance of any supply system subjected to different categories of disturbance events. Moreover, a new mitigation technique through constructing feedback correction signals for disturbance compensation is suggested. For complete automatic on-line monitoring, detection and identification system, a hardware device for disturbance simulation, and a data acquisition card for real time monitoring system were under construction.
\end{abstract}

\section{Key words}

Power quality, disturbance events, DSP, on line monitoring system, and feedback correction signals.

\section{Introduction}

The demand for high quality electricity supply is one of the basic requirements to ensure sustainable economic growth in our increasingly digital-based society. Voltage dips, short interruptions, and voltage harmonics may cause tripping or malfunctioning of protective equipment. These events may be considered one of the most important power quality problems for many industrial customers due to the economic effects that they can have on industrial processes. The development of new methods for real time assessment of voltage events in the distribution systems is of great importance in order to prevent the impact of these disturbances and to evaluate the economics of power quality improvement alternatives.

Several and different methods have been used so far in the literatures to detect and estimate power quality disturbance events [1-3]. The most common method used for this purpose is the calculation of the root mean square (RMS) value of the voltage supply. The main advantage of this method is its simplicity and the main drawback is its dependency on the window length. Depending on the point on the wave when the voltage event starts, the magnitude and duration of the voltage event, and the type of window employed, the RMS magnitude of the voltage supply during the event may be absolutely different.

Wavelet analysis has been used in the last few years as an alternative method for power quality analysis in power systems [4-6]. Different wavelets have been proposed to analyze different types of power quality disturbances. The main drawbacks of wavelet analysis are the difficulty in determining the characteristics as well as the magnitude of disturbance events. Another drawback of wavelet analysis is the difficulty for automatic and real time analysis of power quality disturbances. Moreover, features obtained with wavelet are, still, highly dependent on the type of mother wavelet chosen, which is needed to optimize. Furthermore, the method is applied to power quality records previously obtained by power quality monitors and is not valid for on-line detection and classification of power quality disturbances.

More advanced approaches have been developed for the assessment of the quality of power supply. An expert system has been developed for classification and analysis of voltage dips in terms of their causes [7,8]. Unfortunately, Expert systems were proposed to identify, classify, and diagnose power systems events successfully for a limited number of events [9]. Moreover, Rule based expert systems are highly dependant on if ..then clauses. If many event types or features were analyzed, the expert system would become more complicated.

In recent years, more advanced algorithms for automatic classification were proposed. An on-line power quality disturbance detector was proposed in [10], despite, most of this work is based on wavelet analysis for feature extraction which is not fully agreed by many authors.

According to aforementioned discussion, much effort should be made to enhance power quality assessment methodologies. More sophisticated algorithms for automatic and online detection and classification of 
power quality disturbances should be maintained. The suggested new methodologies should include, automatic monitoring and collecting data from real site measurements, a new and reliable techniques for feature extraction, good estimation and pattern recognition of different types of power quality disturbances, and construct a powerful analysis algorithm for a decision making system in order to enhance the performance of the quality of power supply.

\section{Software Structure Of The Proposed System.}

The proposed method is mainly based on a set of software tools that have been developed using the applications of MatLab6 toolboxes.

\section{A. Distorted Signal Re-construction}

Before any analytical treatment and investigation, distorted signals should be firstly reconstructed to be ready for implementation using the applications of signal processing tools. With the aid of the MatLab toolbox, most of disturbance events were simulated. The simulated signals include the common disturbances such as harmonics, sags, swells, and complete interruption events. Once the status of the quality of power supply that represented by the distorted signal is being ready for further signal processing, a number of powerful software algorithms are then applied and implemented for advanced analysis. A set of powerful computer program codes was introduced for the analysis purposes. The introduced software includes, detection, localization, identification, and quantification processes. The detection/classification process is essentially where the introduced software must have the capability for event recognition and detection. Moreover, the detected events should be categorized according to specific parameters in order to discriminate among different types of disturbance events. The quantification process is needed for assessing the severity caused by each disturbance event to be weighed using indicative numbers for better understanding by both the utility and the customers. Finally, the correction process was suggested using feedback correction signals to improve and enhance the performance of the quality of power supply.

\section{B. Main processes}

The methodology of the proposed method is mainly depending on some of basic, detection, classification, quantification, and compensation processes. The combination of these processes is mainly performed to construct a complete scheme for on-line assessment of the quality of power supply.

In the detection process, Different filters were designed for feature extraction. The designed filters are used to detect power quality variations and to estimate the magnitude and duration of each event type. The designed filters are: Harmonic Filters and RMS voltage variation Filters. Harmonic filters extract all harmonics as well as inter-harmonics coupled with the distorted signal. On the other hand, RMS voltage variation filters are a series of filters that specified by different types of RMS voltage variations and namely sag filter, swell filter, momentary interruption filter,.......etc., These filters have been proposed to detect RMS voltage variations and to estimate the magnitude and the duration of each event type.

The basic principle of the detection process is based on two stages, the scanning stage and the filtering stage. In the scanning stage, the time domain signal is consequently passed through the designed filters for comparison to pre-specified threshold values to detect undesirable records that exceeding the threshold limits. The second stage is the filtering process where any undesirable exceeded value is filtered out from the original disturbance signal leaving the residual signal (waveform after filtering process) free from disturbance events.

In the quantification process, in order for the performance of the distribution networks to be measured or ranked, the effect or the severity of the recorded power quality disturbance phenomena should be quantified in a way that is understandable by both utilities and customers. Most of the used methods for quantifying the severity of the disturbance event are based on extracting qualification numbers from the distorted signal itself. In our work, an alternative method is introduced. The basic idea of the technique is that, instead of the evaluation of event characteristics that extracted from the distorted signal, we can use another basis through the evaluation of disturbance severity to identify more indicative disturbance indices. Based on the introduced idea, indicative factors namely correction factors were suggested to represent event severity upon the quality of power supply. These factors are used to determine how much we need to compensate or correct the amount of distortion that caused by a specified disturbance event and disturbs the supply waveform. For each type of the disturbance events there will be a corresponding correction factor indicating event severity upon the quality of power supply. Moreover, for a supply system subjected to different types and numbers of disturbance events, there will be an overall correction factor indicating, how much should the utility pay to perform an adequate quality supply and to satisfy the customer needs.

Finally, for the compensation process, feedback correction signals were constructed to correct and enhance the performance of the quality of power supply. The addition of such correction signals is mainly to force the distorted signal that monitored on the supply system to be near ideal waveform (pure sine) as possible.

\section{Experimental examples and results.}

The proposed advanced method for power quality assessment is implemented and verified through the examination of detailed case studies. In the case studies the new proposed software algorithms were applied and implemented to investigate variety and different 
simulated distorted signals that undergo different types of disturbance events. Due to limited paper size a generalized case study is introduced. The simulated distorted waveform, used in the case study, includes most of the common disturbance events.

Firstly, a time domain signal is formulated for further investigation as a test example. The signal supposed to be distorted with different categories of disturbance events. Seven cycles of a fully distorted waveform are used to simulate the signal under study. The signal supposed to include the following disturbance phenomena:

- Certain amount of harmonic contents, assumed, the $3^{\text {rd }}$, the $5^{\text {th }}$, the $7^{\text {th }}$, and the $9^{\text {th }}$ harmonic order with magnitudes $0.2,0.3,0.02$, and $0.1 \%$ of the fundamental component respectively.

- Sag events represented by a sudden dip to $0.6 \mathrm{pu}$ voltage value and followed by another dip to 0.4 p.u value with duration $0.014 \mathrm{sec}$ and $0.007 \mathrm{sec}$ cycle respectively.

- Interruption event represented by a sudden change in the rms voltage where the voltage is dropped to 0.0 p.u and persists around $0.014 \mathrm{sec}$.

- Three consequently swell events represented by a sudden increase to $1.4 \mathrm{pu}$ voltage value, $1.1 \mathrm{p} . \mathrm{u}$ value, and $1.6 \mathrm{p} . \mathrm{u}$ value with duration $0.014 \mathrm{sec}, 0.014 \mathrm{sec}$, and $0.028 \mathrm{sec}$ respectively.

Figuers 1, 23, 3 show step by step what is going on, starting from having a pure sinusoidal to the case in which the signal is melted by a set number of disturbance phenomena.

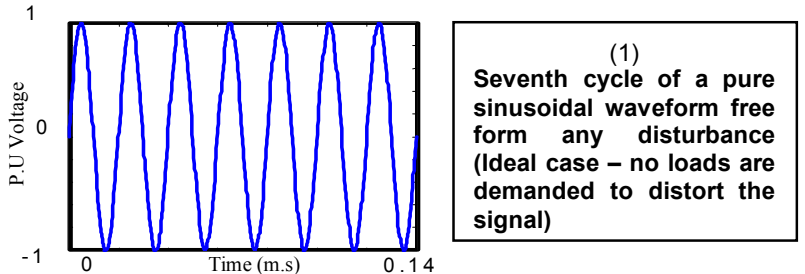

Fig.1 A pure Sinusoidal waveform, No disturbance events are existed

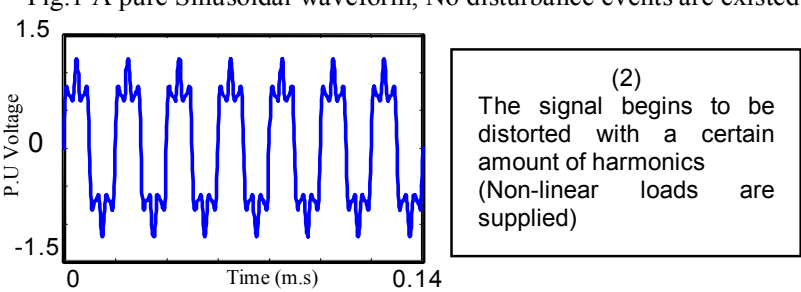

Fig.2 Harmonic disturbance is added to the test signal

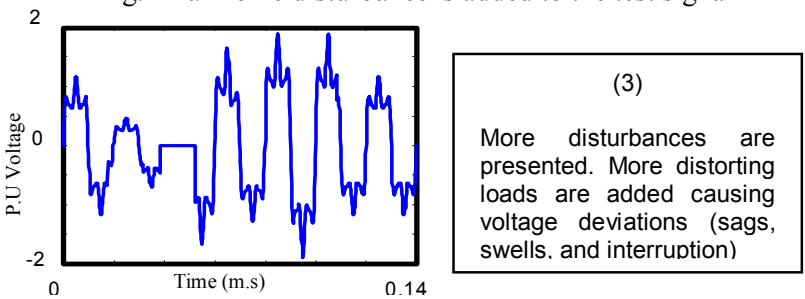

Fig. 3 Rms voltage variation disturbance events are added to the test signal.

The distorted signal (the test signal) is now treated as a time domain signal supposed to be monitored from a bad power quality supply and the main objective now is to extract all disturbance events from the disturbed waveform in order to recognize what types of disturbance phenomena are presented and to quantify severity caused by each disturbance event. Moreover, mitigation techniques should be suggested to enhance the performance of the quality of power supply.

\section{A. Feature extraction (detection process)}

The proposed software algorithm begins with investigating the test signal through the scanning and the filtering processes for detection and extraction all disturbance events supposed to be included in the test signal. Further processes are then take a part in the play to quantify all the detected disturbance phenomena. These procedures are executed as follows:

\section{1) Harmonic Filter is applied to the test signal}

Firstly, the test signal is passed through harmonic filter, the harmonic filter acts to detect harmonic contents and filter them out leaving the test signal pass free of harmonic contents. Fig. 4 shows the process of the sage 1 , harmonic filter acts so as to pickup harmonics from the test signal leaving the test signal suffering, only, RMS voltage variation disturbance phenomena. The filtered data are matched with those previously well known and added previously to the original signal. This gives strong support of the performance of the used software.
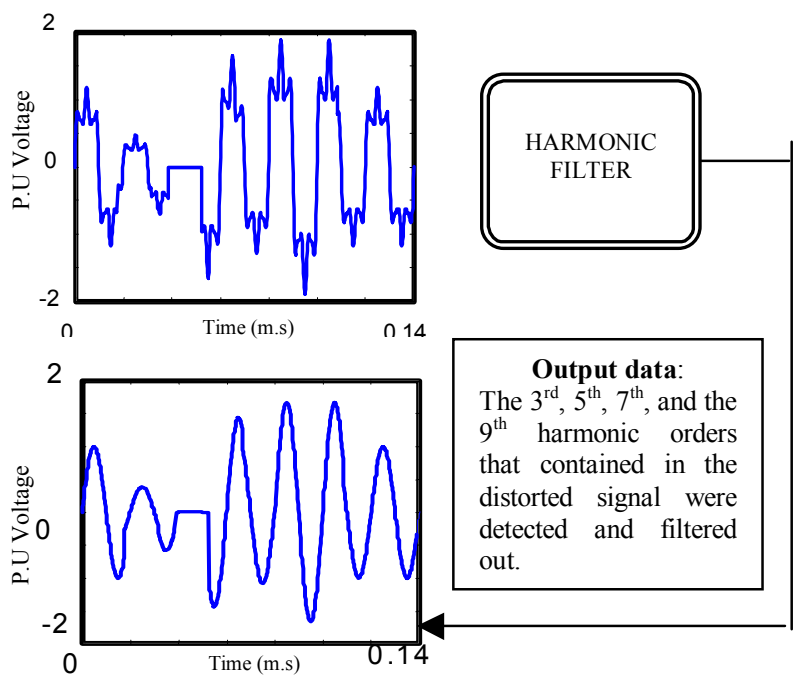

Fig.4 Detection process, stage 1 harmonic disturbances filtering process.

\section{2) RMS Voltage Variations Filter Is Applied To The} Residual Test Signal.

In the second stage, the residual test signal is then passed through the RMS voltage variations filters to detect and identify any RMS voltage variations that violate standard threshold values. The performance of the used filters is to detect the start and the end points of any RMS voltage changes (sag, swell, complete interruption). Once the filtering process is done, all undesirable exceeded values were filtered out from the disturbance signal leaving the residual signal (after filtering process) free of any of the RMS voltage disturbance events. Fig. 5 shows the result 
from the detection process. After the test signal is scanned, different disturbance events were detected and marked by the $(\bullet)$ points, which are signed in the test signal. The $(\bullet)$ points indicate sudden undesirable changes in the RMS voltage variation and determine the start and the end points for each disturbance event detected during the scanning process.

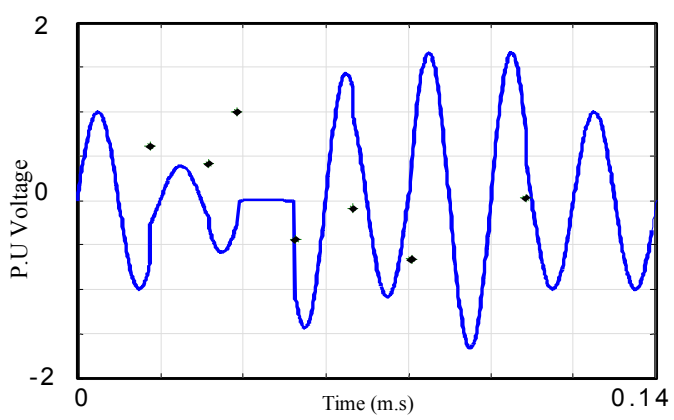

Fig.5 RMS voltage variation detection process, $(\bullet)$ start/ end sign of each detected RMS voltage variation event.

As shown from stage 2, the RMS voltage variations filters succeeded in detecting all of the sudden changes in the RMS voltage magnitudes that violating the threshold limits. The filtered data are matched with those previously well known and added previously to the original signal. The same number of RMS voltage variation disturbance events was detected. Again, this gives strong support for the performance of the used software.

\section{B. Quantification Process (Evaluation disturbance events Correction factors).}

Now, further processes are then applied for quantification purpose. Once the disturbance events are detected, they must be quantified in terms that are understandable for both the utility and customers. As mentioned before, most of the used methods for quantifying disturbance severity upon the quality of power supply are based on extracting the qualification number from the distorted signal itself. In our work, an alternative method is introduced. The basic idea of the technique is that, instead of evaluating the distorted signal itself to extract disturbance indicator, we can use another basis to evaluate disturbance severity that is the correction factors. These factors are used to determine how much we need to compensate or correct the amount of distortion that caused by a specified disturbance event and disturbs the supply waveform. The introduced quantification method was implemented for the test signal in order for quantifying RMS voltage variation disturbance events. Indicative correction factors were calculated and useful results are obtained.

1) Quantification of RMS Voltage Variation Disturbance Events (Evaluation of RMS Voltage Variation Correction Factor)

Most of the methods used for quantifying the RMS voltage variations phenomena are based on the evaluation of the event magnitude and the corresponding event duration. But none of these methods assessed the severity caused as a result of such disturbances. A sag event may perform a deep sag value with a short duration; other may perform a low depth in supply voltage with a long duration. The question is: which event has higher severity? long in depth or long in time? In our proposed work we introduce a factor depends on both, the magnitude as well as the duration. Mathematically, the correction factor can be calculated as indicated in (1).

$C . F=L . O . C \times E . D$

Where: C.F: Correction Factor, L.O.C: Loss Of Cycles (sag or interruption event) or the Rise (swell event) in voltage magnitude that missed or incremented as a result of appropriate events, and E.D: Event Duration.

The introduced idea is implemented to the test signal. Depending on the number of RMS disturbance events, there will be a set of number of correction factors indicating the severity caused by each disturbance event. An indicative diagram namely, event correction diagram is introduced, by which, correction factors can be calculated easily. The correction diagram is a real timemagnitude plot that represents how much does we need to compensate disturbance effects so, it represents the actual severity of the disturbance phenomenon. Furthermore it gives accurate indication of the duration of the event, where there is no missing information during the process of construction. Fig.6 shows the simulated RMS voltage variation time domain signal and the corresponding event correction diagram
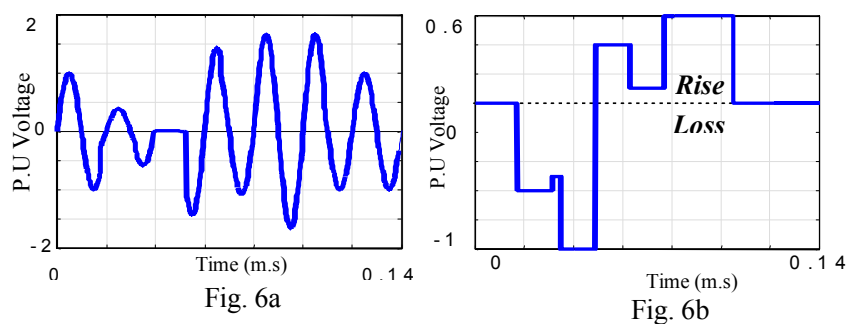

Fig. 6, a- A Test Signal Subjected To A Set Number Of RMS Voltage Disturbance Events And, b- The Corresponding Event Correction Diagram.

From the event correction diagram, indicated by figure $6 . b$, a set number of correction factors were calculated depending on the magnitude and duration coupled with each disturbance event. Moreover an overall correction factor is calculated which can be used for ranking the severity of bad power quality introduced by different supply systems. More indicative results were obtained as shown in Table I. As indicated in Table I. The introduced correction factor will be useful because:

- For different patterns of the same event, the correction factor can be used to discriminate among them.

- The overall correction factor can be useful in ranking the distribution systems so as to recognize the hot feeding points that should be avoided to feed sensitive loads

- For different feeding distribution networks, the overall correction factor can be used for comparing supply system to another. 
TABLE. I Sample Of The Quantification Process Spreadsheet

\begin{tabular}{|c|c|c|c|c|}
\hline $\begin{array}{l}\text { Detected } \\
\text { Event. }\end{array}$ & $\begin{array}{l}\text { Loss / Rise of } \\
\text { voltage value }\end{array}$ & $\begin{array}{c}\text { Event } \\
\text { duration }\end{array}$ & $\begin{array}{l}\text { Event } \\
\text { Type }\end{array}$ & $\begin{array}{c}\text { Correction } \\
\text { Factor }\end{array}$ \\
\hline 1 & 0.6 p.u Loss & $1.4 \mathrm{~m} . \mathrm{sec}$ & Sag & 0.84 \\
\hline 2 & 0.4 p.u Loss & $0.7 \mathrm{~m} . \mathrm{sec}$ & Sag & 0.28 \\
\hline 3 & $\begin{array}{c}\text { Complete P.U } \\
\text { Loss }\end{array}$ & $1.4 \mathrm{~m} . \mathrm{sec}$ & $\begin{array}{l}\text { Interrupti } \\
\text { on }\end{array}$ & 1.4 \\
\hline 4 & 0.4 p.u Rise & $1.4 \mathrm{~m} . \mathrm{sec}$ & Swell & 0.56 \\
\hline 5 & 0.1 p.u Rise & $1.4 \mathrm{~m} . \mathrm{sec}$ & Swell & 0.14 \\
\hline 6 & 0.6 p.u Rise & $2.8 \mathrm{~m} . \mathrm{sec}$ & Swell & 1.68 \\
\hline \multicolumn{4}{|c|}{ Overall Correction Factor $=\sum$ C. $F_{i}$} & 4.9 \\
\hline
\end{tabular}

\section{Disturbance Mitigation process, The Feedback Compensation Signals.}

The basic idea of the mitigation technique is that, how to formulate a compensation signal that can work against the disturbance signal. The addition of such compensation signal should act to cancel the effect of the disturbance signal so that the status of the quality of power supply returns back again innocent/clean as it was before disturbance phenomena has presented in the distribution network.

In the mitigation process, a number of feed back correction signals should be constructed to correct and mitigate the effects of the disturbance events upon the quality of power supply. The compensation signal is constructed so as to compensate the effect appear in the distorted signal where, the addition of such signal to the distorting signal will result to the basic waveform before melted by the disturbance phenomena. For each disturbance event that causes a certain violation in the supply voltage waveform, there will be a feedback correction signal that can correct the disturbance effect and force the supply waveform to be near sinusoidal as possible.

Two types of correction signals are generated, the harmonic compensation signal, and RMS voltage variation compensation signal. Harmonic compensation signal should be injected so as to cancel harmonics that already presented and detected in the system. For example, a harmonic content characterized by $0.2 \mathrm{pu}$ magnitude and with angle in-phase with the fundamental waveform, may be compensated by injected a harmonic signal characterized by the same magnitude but acts outof phase with the fundamental waveform. On the other hand, RMS voltage variation compensation signal should act to restore or damp the loss or the Rise of voltage level that sensed and detected in the supply waveform.

The introduced mitigation technique is implemented using a well-designed computer program. The performance of the computer program is to detectcompensate the disturbance events presented in the supply voltage waveform. For the given test example, two compensation signals were generated for correction purposes, harmonic compensation signal, and RMS voltage variation correction signal. The complete process is well described by the Figs [7-9].
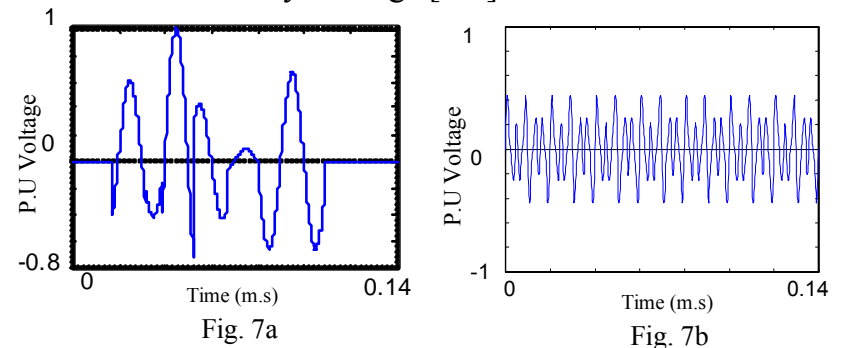

Fig.7. Correction Signals Supposed To Be Added For Compensation, a- RMS Voltage Variation Correction Signal And b- Harmonic Cancellation Signal
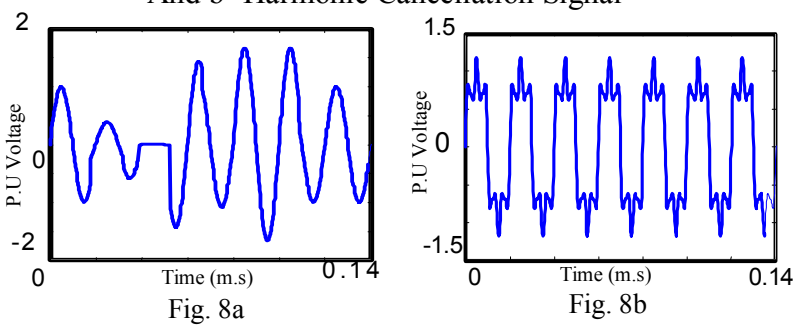

Fig.8. Bad Power Quality Supply Needed to be corrected. aRMS Voltage Variation Disturbance Signal and b- Harmonic Disturbance Signal.

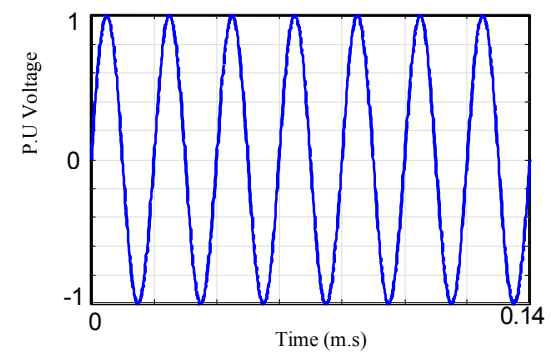

Fig.9. The Resulted Signal After Compensation, Back To Nature Before Melted By The Disturbance Events.

As expected, the addition of such compensation signals were results in obtaining a good power quality supply that represented by a nearly sinusoidal supply waveform.

\section{Conclusion}

A new methodology for on-line detection, classification, quantification, and mitigation system is suggested and implemented using a set of computer algorithms. The introduced system detects accurately any power quality disturbance event and introduces new indicative factors estimating the performance of any supply system subjected to a set of disturbance events. A new mitigation technique through constructing feedback correction signals for disturbance compensation is suggested. For implementation, simulated test examples, software based, of the proposed approaches were addressed. The obtained results indicate high performance of the proposed new method. For complete automatic on-line monitoring, detection and identification system, test experimental examples using the complete hardware devices, real disturbance generator and a data acquisition card for data transfer, are under construction to be presented in the future work. 


\section{References}

[1] David Hat,David uy, Damir Novosel, Steven Kunsman, Carl Lablace, and Marco Tellarini," Improving Power Quality". ABB Review, 4/2000

[2] Emmanouil Styvaktakis, Math Bollen, and Irene $\mathrm{Gu}$,"Automatic Classification of Power System Event Using RMS Voltage Measurements" Submitted for publication in Transactions on Power Delivery

[3] O. Poisson, P. Rioual and M. Meunier, "New Signal Processing Tools Applied to Power Quality Analysis," IEEE Trans. On Power Delivery, vol 14, April 1999.

[4] B. Perunicic, M. Mallini, Z. Wangand Y. Liu, "Power Quality Disturbance Detection And Classification Using Wavelet And Artificial Neural Networks", 8th International Conference on Harmonics and Quality of Power, Vol. 1, 1998, pp. 77-82.

[5] Santoso, Surya, Powers, Edward J., Grady, W. Mack and Hofmann, Peter, "Power Quality Assessment Via Wavelet Transform Analysis," IEEE Trans. On Power Delivery, vol 11, April 1996. pp. 924-930.
[6] Heydt and, A. Galli, "Transient Power Quality Problems Analyzed Using Wavelets". IEEE Trans. On Power Delivery, vol 12, April 19976] E. Styvaktakis, M.H.J.

[7] Bollen and I.Y.H. Gu,2001, "Expert system for Voltage Dip Classification and Analysis," IEEE Power Engineering Socity Summer Meeting, 671-676

[8] E. Styvaktakis, M.H.J. Bollen and I.Y.H. Gu,2002, "Expert system for Classification and Analysis of Power System Events" ,IEEE Trans. On Power Delivery, vol 17, 423-428.

[9] Surya Santoso, Jeff Lamoree, Mack Grady. Edward Powers and Siddharth, "A Scalable PQ Event Identification System", IEEE Trans. On Power Delivery, vol 15, October 2000.

[10] Masoud Karami, Hossien Mokthari and reza Iravani, "Wavelet Based On line Disturbance Detection for Power Quality Applications". IEEE Trans. On Power Delivery, vol 15 , October 2000. 